\title{
EPR-Bell Nonlocality, Lorentz Invariance, and Bohmian Quantum Theory
}

\author{
Karin Berndl ${ }^{\mathrm{a}}$, Detlef Dürr ${ }^{\mathrm{a}}$, Sheldon Goldstein ${ }^{\mathrm{b}}$, Nino Zanghìc \\ a Mathematisches Institut der Universität München, \\ Theresienstraße 39, 80333 München, Germany \\ b Department of Mathematics, Rutgers University, \\ New Brunswick, NJ 08903, USA \\ c Dipartimento di Fisica, Università di Genova, Sezione INFN Genova, \\ Via Dodecaneso 33, 16146 Genova, Italy
}

October 26, 1995

\begin{abstract}
We discuss the problem of finding a Lorentz invariant extension of Bohmian mechanics. Due to the nonlocality of the theory there is (for systems of more than one particle) no obvious way to achieve such an extension. We present a model invariant under a certain limit of Lorentz transformations, a limit retaining the characteristic feature of relativity, the non-existence of absolute time resp. simultaneity. The analysis of this model exemplifies an important property of any Bohmian quantum theory: the quantum equilibrium distribution $\rho=|\psi|^{2}$ cannot simultaneously be realized in all Lorentz frames of reference.
\end{abstract}

\section{Introduction}

Despite the impressive and unquestioned empirical success of quantum theory, the physical meaning of its basic object, the wave function, is still controversial. The standard - or Copenhagen - interpretation of quantum theory asserts that the wave function embodies the most complete description possible of the state of a physical system, while connecting it with experience, and thereby assigning to it physical significance, only via a set of rules for calculating probabilities of results of "measurements." It seems essential within the standard interpretation that "measurements" be distinguished from other physical processes, and that attention be paid to the fact that the theory makes predictions only about results of "measurements": otherwise one runs into the well-known measurement problem or, more pictorially, the paradox of Schrödinger's cat. In any case, the fundamental role of "measurements" (which is sometimes shifted to "observers") in the Copenhagen interpretation leads first of all to the theory's not being well-formulated as a fundamental (as opposed to phenomenological) theory because what constitutes a "measurement" is not specified. Secondly, with regard to cosmology, the necessity to invoke an outside measurement apparatus or observer seems rather awkward. (For extraordinarily clear presentations of the problems of quantum theory as well as of possible solutions see [1, 2, 3].) 
An alternative interpretation resp. theory agreeing with quantum theory on (most of) its predictions which is not based on the notion of "measurement" or "observer" is usually called a "realistic" interpretation resp. theory M More precisely, we shall understand by a "realistic quantum theory" a theory, agreeing with quantum theory on (most of) its predictions, in which it is explicitly specified what the material world is thought to be made of - be it particles or fields or what have you - and how these entities behave. We emphasize that this by no means implies a "naive realism"; on the contrary, these entitieswhat Bell called the "beables" of the theory - can be rather remote from our perception of the world. Moreover, the performance of experiments may disturb the behavior of the beables, so that the "observed" properties of matter may be quite different from those left "unobserved."

In nonrelativistic quantum theory there are two principal routes for setting up a realistic quantum theory: Either the wave function is not the complete description of the state of a physical system, but must be supplemented by some further quantities, commonly (and unfortunately) called "hidden variables," or the unitary evolution of the wave function must be modified. The paradigmatic example of the first route is Bohmian mechanics [5, 6], that of the second route the theory of Ghirardi, Rimini, and Weber (GRW) [7]. We shall call a realistic quantum theory of the first kind a "Bohmian theory." Our objective is to find a Lorentz invariant Bohmian theory which extends Bohmian mechanics, i.e., which leads to Bohmian mechanics in the nonrelativistic limit.

For systems of a single particle, a Lorentz invariant Bohmian theory is immediately specified [8, 9, 10]: the beables are the wave function $\psi\left(x^{\mu}\right)$ and a particle path, which may be specified as an integral curve of a 4-vector field $j^{\mu}$ (for example, of the current naturally associated with the Klein-Gordon or Dirac wave function)

$$
\frac{d X^{\mu}}{d s}=j^{\mu}\left(X^{\mu}\right)
$$

Multiplication of $j^{\mu}$ by a positive scalar field $a\left(x^{\mu}\right)$ changes only the parametrization, not the path, understood as the equivalence class of curves $X^{\mu}: \mathbb{R} \rightarrow \mathbb{R}^{4}$, $s \mapsto X^{\mu}(s)$ differing only in their parametrization, or as the image $X^{\mu}(\mathbb{R})$ of a curve $X^{\mu}$, i.e., a 1-dimensional subset of $\mathbb{R}^{4}$. If $j^{\mu}$ is everywhere timelike, i.e., if $j_{\mu} j^{\mu}>0$ with the sign convention for the metric $g_{00}=1, g_{11}=g_{22}=g_{33}=-1$, a parametrization by proper time may be obtained by replacing Eqn. (1) by $\frac{d X^{\mu}}{d \tau}=u^{\mu}\left(X^{\mu}\right)$ with the 4 -velocity $u=a j, a=\left(j_{\mu} j^{\mu}\right)^{-1 / 2}$. In general there is no distinguished parametrization, and the parametrization chosen in writing Eqn. (11) has no physical significance as such: all equations of the form $\frac{d X^{\mu}}{d s}=a\left(X^{\mu}\right) j^{\mu}\left(X^{\mu}\right)$ with different $a$ are physically equivalent.

The Dirac current $j^{\mu}=\bar{\psi} \gamma^{\mu} \psi$ is a timelike future-oriented vector; thus the curves which are solutions of (四) run from $t=-\infty$ to $t=+\infty$, never

\footnotetext{
${ }^{1}$ This is a rather unfortunate term - can "realism," i.e., the belief that there is a material world the description of which is the task of physics, seriously be questioned in physics? See also 那.
} 
backwards in time, with velocity everywhere bounded by $c$. In particular, every path crosses every $t=$ const.-hyperplane of every Lorentz frame of reference - or, indeed, every spacelike hypersurface - exactly once, and thus there is a one-toone correspondence between paths and points - their crossing points - on an arbitrary spacelike hypersurface.

Because the Dirac current is divergence free, it allows moreover for a straightforward introduction of a dynamically distinguished measure on the set of particle paths as follows: In an arbitrary Lorentz frame, take $\rho=j^{0}=\psi^{\dagger} \psi$ as the density of crossings through a $t=t_{0}$-hyperplane at an arbitrary time $t=t_{0}$. Then the density of crossings $\rho$ arising from (11) satisfies $\rho=j^{0}$ at all times in this frame, i.e., $j^{0}=\psi^{\dagger} \psi$ is an "equivariant" density. Furthermore, "quantum equilibrium" $\rho=j^{0}$ holds then in all Lorentz frames at all times. The distribution $\rho=j^{0}=\psi^{\dagger} \psi$ is hence the relativistic generalization of the "quantum equilibrium distribution" $\rho=|\psi|^{2}$ of nonrelativistic Bohmian mechanics, which is the essential tool for the derivation of the nonrelativistic quantum formalism [6].

In fact, any divergence free current $j^{\mu}$, in particular also the Klein-Gordon current which is in general not globally timelike, gives rise to a natural measure on the set of trajectories which are integral curves of $j^{\mu}$ (i.e., solutions of (1)), in a way extending the above definition of a natural measure for the BohmDirac theory. Moreover, the fact that Klein-Gordon trajectories possibly "run backwards in time" may well be viewed as naturally describing pair creation and annihilation. We shall discuss these topics in a subsequent work.

For systems of more than one particle, it is not at all obvious how to construct a Lorentz invariant realistic quantum theory, in fact it is not even clear whether this is possible at all. The problem is due to the unavoidable nonlocality of any realistic (or, more accurately, of any precise ([1], pp. 171, 194)) version of quantum theory: The incompleteness argument of Einstein, Podolsky, and Rosen (EPR) 11] together with the analysis of Bell ([1], Chapter 2) 5 shows that every theory giving the quantum mechanical predictions must be nonlocal. This obviously conflicts with what is often considered to be the essence of Einsteinian relativity - the locality of physical interactions. The requirement of the Lorentz invariance of a physical theory, however, doesn't force locality. Thus a nonlocal Lorentz invariant theory is certainly possible. This is already rather clear from the meaning of the terms: While "Lorentz invariance" describes the behavior of a theory under certain transformations of reference frame, the term "locality" conveys that there is no action-at-a-distance. For an exhaustive discussion, see [12]. An interesting classical example is the action-ata-distance theory of Schwarzschild-Tetrode-Fokker-Wheeler-Feynman (see 13] and the references therein) replacing classical electrodynamics: In this Lorentz invariant theory the point charges interact directly with each other (on forward and backward light cones) - in a manner unmediated by an electromagnetic field, which is not a fundamental entity here.

\footnotetext{
${ }^{2}$ If $\int j^{0} d x^{1} d x^{2} d x^{3}<\infty$, we may normalize the measure by replacing $j$ by $a j$ with $a^{-1}=$ $\int j^{0} d x^{1} d x^{2} d x^{3}$ to obtain a probability measure.

${ }^{3}$ For particularly clear presentations see also [⿰], Chapter 16, as well as [3, 12 .
} 
Bohmian mechanics [5, 6] is manifestly nonlocal: the velocity of a particle at time $t$ depends in general upon the positions of all the other particles at that time

$$
\mathbf{v}_{k}\left(\mathbf{q}_{1}, \ldots, \mathbf{q}_{N}, t\right)=\frac{\hbar}{m_{k}} \operatorname{Im} \frac{\nabla_{k} \psi_{t}\left(\mathbf{q}_{1}, \ldots, \mathbf{q}_{N}\right)}{\psi_{t}\left(\mathbf{q}_{1}, \ldots, \mathbf{q}_{N}\right)} .
$$

In contrast to Newtonian mechanics, where for realistic interactions the instantaneous influence of the other particles decreases with increasing distance, and therefore widely separated systems are (in an certain sense) approximately independent, for Bohmian mechanics the spatial distance between the particles is irrelevant so long as the wave function of the entire system has a suitably entangled form.

For a system of many Dirac particles, Bohm [14, 9] has proposed the following guiding condition

$$
\mathbf{v}_{k}=\frac{\psi^{\dagger} \boldsymbol{\alpha}_{k} \psi}{\psi^{\dagger} \psi}
$$

which is formulated with respect to a certain reference frame, and is in fact not Lorentz invariant. Analogously to the nonrelativistic theory, the quantum flux equation which is a consequence of the many-particle Dirac equation guarantees that $\psi^{\dagger} \psi$ is an equivariant ensemble density for this dynamical system in the chosen reference frame, and therefore this theory reproduces the quantum predictions insofar as they derive from the probability density $\psi^{\dagger} \psi$. These predictions don't contain a trace of the preferred frame: Lorentz invariance holds on the observational, but not on the fundamental level. (The situation is similar for Bohm's quantum field theory [5, 14, 91.)

There have been a number of arguments to the effect that a Bohmian theory must involve a preferred frame of reference, and thus must violate Lorentz invariance. The most interesting such argument has been put forward by Hardy [15], who by discussing an intriguing experiment - one that we shall discuss in this paper as well, and that has been shown to contain even more surprises ([16, 17], and in particular a nonlocality argument in a sense involving but one photon [18]) - claims to have shown that every realistic quantum theory must possess a preferred frame of reference, and thus that there can be no Lorentz invariant realistic quantum theory.

However, because it rests on an unsuitable "reality criterion" [19, 20], Hardy's argument is wrong. There are even counterexamples to Hardy's argument: the multitime translation invariant formulation of the GRW theory by Bell ( [1], Chapter 22) as well as the multitime translation invariant Bohmian theory we present in this paper are realistic models for the discussed experiment without a preferred frame. Furthermore, there is an outline for a relativistic Bohmian quantum field theory, in which a foliation of space-time into spacelike hypersurfaces is an additional beable 21]. Finally one can find a number of models of relativistic $N$-particle theories with an action-at-a-distance defined in a Lorentz invariant manner, models that therefore have the potential to properly and relativistically describe quantum nonlocality as exhibited in Hardy's experiment. We allude to one such possibility in Section 1 , but shall discuss these models in a subsequent work. No nontrivial Lorentz invariant realistic quantum 
theory is as yet known, but there is no compelling argument that this should be impossible. T On the contrary, the above mentioned models are steps towards a Lorentz invariant realistic quantum theory. One should, however, be aware that the determination of the empirical predictions of these models may present a difficult problem; in fact, for many models there is in general no reason that quantum equilibrium should hold with respect to any reasonable family of hypersurfaces; thus the statistical analysis will be different from that in nonrelativistic Bohmian mechanics and moreover, presumably, the predictions of such a theory won't agree with (all of) those of quantum theory.

Similarly Albert ([3], p. 159ff), Bohm and Hiley ([9], Section 12.6), Ghirardi et al. 22], and Hardy and Squires [23] also argue that a Bohmian theory must violate Lorentz invariance because a preferred frame is needed. The above mentioned models without a preferred frame (but with some "simultaneity" fixed in a Lorentz invariant way-note that this entails that there always are Lorentz frames in which future events influence the past, in contrast to assumptions in [3, 9, 22, 23]) show that less is established than claimed.

This paper is organized as follows: We show in Section that the joint distribution of the particle positions cannot in general agree with the quantum mechanical distribution in all Lorentz frames. This is in contrast to the situation for 1 particle - or, indeed, $N$ independent particles - as explained above. We also discuss why nevertheless the quantum mechanical predictions for performed measurements can be obtained. In Section 3 we present a concrete step towards a Lorentz invariant Bohmian theory: a Bohmian theory invariant under certain limits of Lorentz transformations, limits defining a symmetry that expresses the essence of relativistic space-time - the non-existence of absolute time resp. simultaneity. These transformations, which we shall call "multitime translations," have been discussed by Bell in connection with the GRW theory ([1]], Chapter 22, and [24]; Bell calls them "relative time translations"). In Section 3.1 we describe a multitime translation invariant formulation of Schrödinger's equation for systems composed of noninteracting parts. In Section 3.2 we present the corresponding multitime translation invariant Bohmian theory and discuss its statistical properties. In Section 3.3 we apply the general analysis to Hardy's experiment, focusing on how this experiment illustrates the general discussion in Section 2 .

We remark that there is no difficulty formulating a Lorentz invariant multitime version of the Dirac equation for a system of noninteracting Dirac particles [25]. However, the corresponding Lorentz invariant Bohmian theory lacks statistical transparency. Indeed, at first sight, Lorentz invariance and statistical transparency appear to be mutually exclusive. See Section t for a bit more detail on this, as well as some further reflections on Lorentz invariance.

For systems that consist of noninteracting subsystems, Bell has shown that the GRW theory can be reformulated in such a way that it becomes invariant

\footnotetext{
${ }^{4}$ And the history of the issue of hidden variables, i.e., of the completeness of the description provided by the wave function, should strongly warn us against too readily accepting impossibility claims.
} 
under multitime translation ([1], Chapter 22). He regarded this as an important step towards a genuinely Lorentz invariant precise formulation of quantum theory, declaring that "And I am particularly struck by the fact that the model is as Lorentz invariant as it could be in the nonrelativistic version. It takes away the ground of my fear that any exact formulation of quantum mechanics must conflict with fundamental Lorentz invariance." ([四], p. 209). The multitime translation invariant Bohmian theory we discuss in this paper may, perhaps, be regarded as showing that this assertion applies also to Bohmian mechanics.

For simplicity, we shall put all masses $m_{k}=1$ and $\hbar=c=1$.

\section{Quantum equilibrium cannot hold in all Lorentz frames}

We consider an arbitrary theory for $N(\geq 2)$ particles, i.e., a (possibly statistical) specification of all possible $N$-tuples of space-time paths for the $N$ particles (for example as given by solutions of a system of differential equations). We shall call each such possible "history" an $N$-path. We assume that each spacelike hypersurface is crossed exactly once by each trajectory, and consider an arbitrary probability measure $P$ on the $N$-paths. This determines the distribution of crossings $\rho^{\Sigma}: \Sigma^{N} \rightarrow \mathbb{R}$ for any spacelike hypersurface $\Sigma$.

We now want the probabilistic predictions of the theory to agree as far as possible with those of quantum theory. Complete agreement would be straightforward if for any quantum state $\psi$ there were a $P$ such that for all spacelike hypersurfaces $\Sigma$ the distribution of crossings $\rho^{\Sigma}$ agrees with the quantum mechanical joint distribution of the (measured) positions on $\Sigma$. For $\Sigma$ a spacelike hyperplane, i.e., a simultaneity plane or constant-time slice of a Lorentz frame $\Lambda$, this is given by $\left|\psi^{\Sigma}\right|^{2}$ where $\psi^{\Sigma}=\psi^{\Lambda}$, the wave function in frame $\Lambda$. However, this is not in general possible:

(*) There does not in general exist a probability measure $P$ on $N$-paths for which the distribution of crossings $\rho^{\Sigma}$ agrees with the corresponding quantum mechanical distribution on all spacelike hyperplanes $\Sigma$.

The field theoretical analogue of this assertion has been conjectured by Dürr, Goldstein, and Zanghì in 1990 [21]. Samols discusses the equivalent result for his stochastic realistic model of a light cone lattice quantum field theory [26].

The caveat "in general" refers to the fact that there are exceptional physical situations for which such a $P$ does exist. Consider, for example, 2 independent Dirac particles, i.e., with a wave function that is a product of 1-particle wave functions $\psi=\psi_{a} \psi_{b}$ and independent evolutions given by (11): $\frac{d X_{k}}{d s}=j_{k}\left(X_{k}\right)$, $j_{k}^{\mu}=\bar{\psi}_{k} \gamma^{\mu} \psi_{k}, k=a, b$. Then, as explained above, if $\rho^{\Sigma_{0}}=j_{a}^{0} j_{b}^{0}$ with respect to one spacelike hyperplane $\Sigma_{0}$, then $\rho^{\Sigma}=j_{a}^{0} j_{b}^{0}$ for all spacelike hyperplanes $\Sigma$. We believe, however, that such exceptional physical situations are rare.

The assertion $(*)$ is more or less an immediate consequence of any of the no-hidden-variables-nonlocality theorems-Bell's [1], that of Clauser, Horne, 
Shimony, Holt [27], that of Greenberger, Horne, Zeilinger [28] (see also [29]), or what have you - for the spin components of a multiparticle system: By means of a suitable placement of appropriate Stern-Gerlach magnets the inconsistent joint spin correlations can be transformed to (the same) inconsistent joint spatial correlations for particles at different times. Since the existence of a probability measure $P$ on $N$-paths implies the existence and hence the consistency of all crossing distributions, the assertion follows.

Since this this is an important result, we shall provide an elaboration using one of the sharpest nonlocality theorems, that of Hardy 15]. It should be clear from our treatment of this example how to arrange the magnets to deal with any other version.

Consider the experiment described in Figure 1, which is similar to the EPRBohm experiment and which is a slight modification of the experiment discussed by Hardy [15], which we shall call "Hardy's experiment." A pair of particles is prepared in Hardy's state $\psi=\psi_{\text {Hardy }}$, which has, say in frame $I$, the form (we write only the "spin" part)

$$
\begin{aligned}
\psi_{\text {Hardy }} & =\frac{1}{\sqrt{3}}\left(|+\rangle_{z}^{a}|-\rangle_{z}^{b}-\sqrt{2}|-\rangle_{x}^{a}|+\rangle_{z}^{b}\right) \\
& =\frac{1}{\sqrt{3}}\left(|-\rangle_{z}^{a}|+\rangle_{z}^{b}-\sqrt{2}|+\rangle_{z}^{a}|-\rangle_{x}^{b}\right) \\
& =\frac{1}{\sqrt{3}}\left(|+\rangle_{z}^{a}|-\rangle_{z}^{b}-|+\rangle_{z}^{a}|+\rangle_{z}^{b}+|-\rangle_{z}^{a}|+\rangle_{z}^{b}\right) \\
& =\frac{1}{\sqrt{12}}\left(|+\rangle_{x}^{a}|+\rangle_{x}^{b}-|+\rangle_{x}^{a}|-\rangle_{x}^{b}-|-\rangle_{x}^{a}|+\rangle_{x}^{b}-3|-\rangle_{x}^{a}|-\rangle_{x}^{b}\right)
\end{aligned}
$$

where $|+\rangle_{x},|-\rangle_{x}$ denote the eigenfunctions of $\sigma_{x}$ with eigenvalue +1 resp. -1 , and $|+\rangle_{z},|-\rangle_{z}$ denote the eigenfunctions of $\sigma_{z}$ with eigenvalue +1 resp. -1 . We have used that $|+\rangle_{x}=\left(|+\rangle_{z}+|-\rangle_{z}\right) / \sqrt{2}$ and $|-\rangle_{x}=\left(|+\rangle_{z}-|-\rangle_{z}\right) / \sqrt{2}$. Denoting by $(a, b)_{(x, z)}$ the components of spin in direction $x$ resp. $z$ of particle $a$ resp. $b$, the following quantum mechanical predictions can be read off from the form of the wave function:

$$
\begin{array}{cc}
a_{x}=+1 \Rightarrow b_{z}=-1 & (\text { from (4)) } \\
b_{x}=+1 \Rightarrow a_{z}=-1 & (\text { from (5)) } \\
\operatorname{not}\left(a_{z}=-1 \text { and } b_{z}=-1\right) & (\text { from (6) }) \\
\operatorname{Prob}\left(a_{x}=+1 \text { and } b_{x}=+1\right)=\frac{1}{12} & (\text { from (7) })
\end{array}
$$

These predictions are clearly inconsistent for random variables since the last one together with the first two then imply that $\left\{a_{z}=-1\right.$ and $\left.b_{z}=-1\right\}$ has probability at least $1 / 12$.

Now suppose that the setup is such that after the two particles are widely separated from each other, each of them runs through a Stern-Gerlach magnet $A_{x}$ resp. $B_{x}$, which splits the respective parts of the wave function into the eigenfunctions $|+\rangle_{x}^{a}$ and $|-\rangle_{x}^{a}$ resp. $|+\rangle_{x}^{b}$ and $|-\rangle_{x}^{b}$. These parts are later recombined by reverse magnets after which they are lead through a second Stern-Gerlach magnet $A_{z}$ resp. $B_{z}$, which splits the wave function into the 


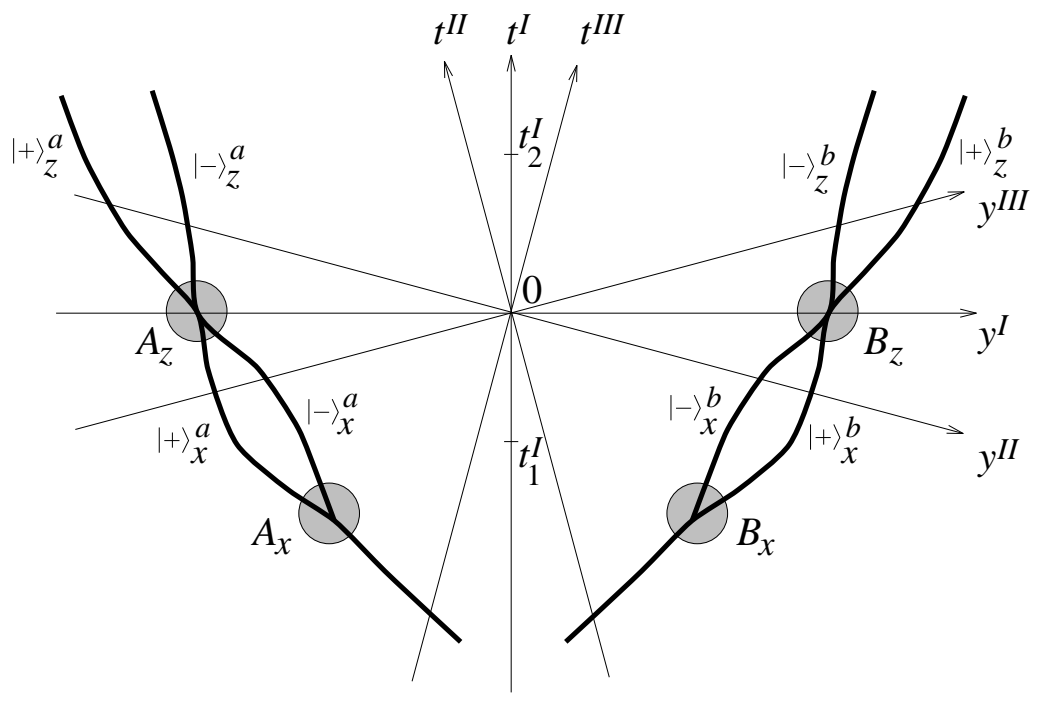

Figure 1: Space-time diagram of the evolution of the wave function in Hardy's experiment. In the shaded regions there are Stern-Gerlach magnets $A_{x}, A_{z}, B_{x}$, and $B_{z}$, which split the respective parts of the wave function into the respective eigenfunctions $(|+\rangle,|-\rangle)_{x, z}^{a, b}$. Three different frames of reference are also drawn.

eigenfunctions $|+\rangle_{z}^{a}$ and $|-\rangle_{z}^{a}$ resp. $|+\rangle_{z}^{b}$ and $|-\rangle_{z}^{b}$. Thus the spin components are (more or less) perfectly correlated with the path variables as indicated in Figure 1, which therefore inherit the inconsistency of the spin components. The assertion follows.

We remark that the measurements to which the quantum mechanical predictions refer might well be performed in this way, but with the insertion of photographic plates behind the appropriate Stern-Gerlach magnets.

We perhaps should be even more explicit, particularly since we will need later to refer to some of the notation to be developed here. Suppose that there is a theory for 2 particles for which the distributions of crossings $\rho^{\Sigma}$ agrees with the quantum mechanical distribution for position (measurements), given by $\left|\psi^{\Sigma}\right|^{2}$, for all spacelike hyperplanes $\Sigma$. The hyperplanes we shall consider are simultaneity planes in the Lorentz frames $I$ at $t^{I}=t_{1}^{I}$ and $t_{2}^{I}, I I$ at $t^{I I}=0$, and $I I I$ at $t^{I I I}=0$, as shown in Figure 11. We shall denote these by $\Sigma^{I}\left(t_{1}^{I}\right), \Sigma^{I I}(0)$, etc.. Furthermore we shall abbreviate $\psi^{\Sigma^{I}\left(t_{1}^{I}\right)}$ by $\psi_{1}^{I}, \rho^{\Sigma^{I}\left(t_{1}^{I}\right)}$ by $\rho_{1}^{I}, \psi^{\Sigma^{I I}(0)}$ by $\psi_{0}^{I I}, \rho^{\Sigma^{I I}(0)}$ by $\rho_{0}^{I I}$, etc..

Consider the configurational part of the wave function in these Lorentz frames. We shall now regard $| \pm\rangle_{x, z}^{a, b}$ as representing the appropriate configurational part of the wave function as indicated in Figure 1, with $\operatorname{supp}| \pm\rangle_{x, z}^{a, b}$ 
denoting its spatial support.] Then

$$
\begin{aligned}
\psi_{1}^{I} & =\frac{1}{\sqrt{12}}\left(|+\rangle_{x}^{a}|+\rangle_{x}^{b}-|+\rangle_{x}^{a}|-\rangle_{x}^{b}-|-\rangle_{x}^{a}|+\rangle_{x}^{b}-3|-\rangle_{x}^{a}|-\rangle_{x}^{b}\right) \\
\psi_{0}^{I I} & =\frac{1}{\sqrt{6}}\left(|-\rangle_{z}^{a}|+\rangle_{x}^{b}+|-\rangle_{z}^{a}|-\rangle_{x}^{b}-2|+\rangle_{z}^{a}|-\rangle_{x}^{b}\right) \\
\psi_{0}^{I I I} & =\frac{1}{\sqrt{6}}\left(|+\rangle_{x}^{a}|-\rangle_{z}^{b}+|-\rangle_{x}^{a}|-\rangle_{z}^{b}-2|-\rangle_{x}^{a}|+\rangle_{z}^{b}\right) \\
\psi_{2}^{I} & =\frac{1}{\sqrt{3}}\left(|+\rangle_{z}^{a}|-\rangle_{z}^{b}-|+\rangle_{z}^{a}|+\rangle_{z}^{b}+|-\rangle_{z}^{a}|+\rangle_{z}^{b}\right)
\end{aligned}
$$

From the assumption that $\rho^{\Sigma}=\left|\psi^{\Sigma}\right|^{2}$ in all frames, we obtain, from (12) or (11), that for the simultaneity surface $\Sigma=\Sigma^{I}\left(t_{1}^{I}\right)$

$$
\begin{aligned}
& \int_{\operatorname{supp}|+\rangle_{x}^{a} \times \operatorname{supp}|+\rangle_{x}^{b}} \rho_{1}^{I}\left(q_{a}, q_{b}\right) d q_{a} d q_{b} \\
= & \int_{\operatorname{supp}|+\rangle_{x}^{a} \times \operatorname{supp}|+\rangle_{x}^{b}}\left|\psi_{1}^{I}\left(q_{a}, q_{b}\right)\right|^{2} d q_{a} d q_{b}=\frac{1}{12} .
\end{aligned}
$$

For the simultaneity surfaces $\Sigma^{I I}(0)$ and $\Sigma^{I I I}(0)$ we have from (13) resp. (14) (or (9) resp. (8)) that

$$
\begin{array}{cll}
\rho_{0}^{I I}\left(q_{a}, q_{b}\right)=0 & \text { for } & \left(q_{a}, q_{b}\right) \in \operatorname{supp}|+\rangle_{z}^{a} \times \operatorname{supp}|+\rangle_{x}^{b}, \\
\rho_{0}^{I I I}\left(q_{a}, q_{b}\right)=0 & \text { for } \quad & \left(q_{a}, q_{b}\right) \in \operatorname{supp}|+\rangle_{x}^{a} \times \operatorname{supp}|+\rangle_{z}^{b},
\end{array}
$$

and for $\Sigma=\Sigma^{I}\left(t_{2}^{I}\right)$ from (15) or (10) that

$$
\rho_{2}^{I}\left(q_{a}, q_{b}\right)=0 \quad \text { for } \quad\left(q_{a}, q_{b}\right) \in \operatorname{supp}|-\rangle_{z}^{a} \times \operatorname{supp}|-\rangle_{z}^{b}
$$

Consider now that part of the ensemble of two-paths containing paths that cross supp $|+\rangle_{x}^{a} \times \operatorname{supp}|+\rangle_{x}^{b}$. From (16), this has probability 1/12. From (17), particle $a$ will be in $\operatorname{supp}|-\rangle_{z}^{a}$ at $t_{2}^{I}$; from (18), particle $b$ will be in $\operatorname{supp}|-\rangle_{z}^{b}$ at $t_{2}^{I}$; thus

$$
\int_{\operatorname{supp}|-\rangle_{z}^{a} \times \operatorname{supp}|-\rangle_{z}^{b}} \rho_{2}^{I}\left(q_{a}, q_{b}\right) d q_{a} d q_{b} \geq \frac{1}{12},
$$

in contradiction with (19). (This argument assumes that, say, the crossing track of particle $a$ for $\Sigma^{I}\left(t_{2}^{I}\right)$ agrees with that for $\Sigma^{I I}(0)$-i.e., that there is no sudden change of track. By a suitable choice of geometry the violation of this assumption can be made as implausible as we like.)

We can more briefly, though somewhat imprecisely, rephrase $(*)$ by saying that "Quantum equilibrium $\left(\rho=|\psi|^{2}\right)$ cannot hold in all Lorentz frames." Although the notions of the wave function $\psi$ in position representation as well

\footnotetext{
${ }^{5}$ It should perhaps be noted that a Dirac spinor which in frame $I$ is a spin $x / z$ eigenfunction will not be a spin $x / z$ eigenfunction in the frames $I I$ or $I I I$ which are boosted in the $y$-direction. Our notation here should not be construed as implying otherwise.
} 
as that of a position measurement are problematical in relativistic quantum theory, the impact of this statement is not thereby diminished. In fact, the statement " $\rho=|\psi|^{2}$ cannot hold in all Lorentz frames" should be understood as follows: The joint distributions given by quantum theory for position measurements (from whatever formalism they arise) cannot in general agree with the distribution of the actual particle positions in all Lorentz frames. This is the case, as pointed out above, already if only the (experimentally well-established) predictions of the distribution of spin measurements - spin is measured, as is any observable, cf. [30], ultimately by measuring some position in a suitable experiment (here with Stern-Gerlach magnets) - in the singlet state (which is the relevant state for the earlier versions of the nonlocality theorems) are considered.

An immediate question is whether this leads, for a theory with trajectories, to experimentally detectable violations of quantum mechanical predictions. That it ain't necessarily so will be illustrated by a concrete model in a later section. But it is already clear from (nonrelativistic) Bohmian mechanics that the validity of $\rho=|\psi|^{2}$ in just one frame is sufficient to derive the quantum mechanical predictions for observations at different times: Assume that the frame corresponding to (Newtonian) absolute time - the frame in which quantum equilibrium $\rho=|\psi|^{2}$ holds for Bohmian mechanics - corresponds to system $I$ in Hardy's experiment in Figure 1. To derive from Bohmian mechanics the correct prediction for the joint distribution of a measurement of $a_{x}$ and a later measurement of $b_{z}$, one has to take into account that the actual performance of measuring $a_{x}$, which requires an intervention such as the suitable insertion of a photographic plate, influences the future evolution of the whole system, and in particular, nonlocally and instantaneously, the future path of particle $b$. This can be conveniently described in terms of the effective "collapse of the wave function." The "unmeasured" distributions do not in general give the correct predictions for the outcomes of experiments! For a rather detailed discussion of related matters, see [6], Sections 8-10.

Moreover, it is rather clear that any two theories agreeing at all times on the spatial distribution of particles for some frame must be empirically equivalent, though we shall not try here to give a precise formulation of this assertion. We note, however, that for a theory involving a foliation of space-time into hypersurfaces, such as the proposal of Dürr, Goldstein, and Zanghi [21], as well as that of Samols [26], it is natural to demand that "quantum equilibrium" hold on these hypersurfaces. For the proposal in section 3.2 of this paper, a theory involving particle interactions that are instantaneous with respect to a specified synchronization, one is lead to demand "quantum equilibrium" with respect to this synchronization. That this indeed suffices to recover the quantum mechanical predictions for the outcomes of all joint measurements is implied by the fact that the joint results for any family of measurements can always be transferred to a common place and time - and must be if these results are to be subject to the analysis of a single individual (cf. [21, 26], and [6], point 19 on p. 900). This suggests that even a suitable kind of "local quantum equilibrium" should be sufficient to obtain the standard quantum mechanical predictions. 


\section{The multitime formalism}

\subsection{Multitime translation invariance}

Consider a system composed of $n$-we put $n=2$ for simplicity-widely separated subsystems. Even observers who are slowly ("nonrelativistically") moving relative to each other need not agree on the simultaneity of events in the separated subsystems: let $\left(t_{\alpha}, x_{\alpha}\right),\left(t_{\beta}, x_{\beta}\right)$ be the coordinates of the events $\alpha$ resp. $\beta$ for observer 1 . We may put $t_{\alpha}=0, x_{\alpha}=0$. The two events are simultaneous, $t_{\alpha}=t_{\beta}$, and widely separated from each other, $x_{\beta} \gg 1$. A second observer, slowly moving in the $x$-direction relative to the first observer, will describe the same events by the following primed coordinates, cf. Figure 2:

$$
\begin{aligned}
& t_{\alpha}^{\prime}=t_{\alpha}=0, x_{\alpha}^{\prime}=x_{\alpha}=0, \\
& t_{\beta}^{\prime}=\gamma\left(t_{\beta}-v x_{\beta}\right) \approx-\vartheta, \quad x_{\beta}^{\prime}=\gamma\left(x_{\beta}-v t_{\beta}\right) \approx x_{\beta},
\end{aligned}
$$

where $v \approx 0$, so that $\gamma=1 / \sqrt{1-v^{2}} \approx 1$. It is further assumed that $x_{\beta}$ is sufficiently large that $v x_{\beta}=\vartheta$ is of order unity. For observer 2, the events $\alpha$ and $\beta$ are not simultaneous, $t_{\alpha}^{\prime} \neq t_{\beta}^{\prime}$, not even approximately. More precisely, in the limit in which $x_{\beta} \rightarrow \infty$ and $v \rightarrow 0$ in such a manner that $v x_{\beta}=$ $\vartheta \neq 0$, the Lorentz transformation becomes simply a translation of relative time. Consequently, for the case of a system composed of widely separated subsystems we might demand of a nonrelativistic theory invariance with respect to independent shifts of the zeros of the subsystems' time scales (on subsystem clocks). The relevance of this nonrelativistic residue, or analogue, of Lorentz invariance, especially for the discussion of the possibility of a Lorentz invariant realistic quantum theory, has been pointed out by Bell ([1], Chapter 22, and [24]).

To specify the space-time transformation corresponding to this change in frame of reference, we have to introduce two separate coordinate systems for the two widely separated subsystems $a$ and $b$. On configuration-space-time, the multitime translation is given by

$$
\begin{aligned}
L_{\tau}: \mathbb{R} \times \mathbb{R}^{3 N_{a}} \times \mathbb{R} \times \mathbb{R}^{3 N_{b}} & \longrightarrow \mathbb{R} \times \mathbb{R}^{3 N_{a}} \times \mathbb{R} \times \mathbb{R}^{3 N_{b}}, \tau=\left(\tau_{a}, \tau_{b}\right) \in \mathbb{R}^{2} \\
z:=\left(z_{a}, z_{b}\right):=\left(t_{a}, q_{a}, t_{b}, q_{b}\right) & \longmapsto\left(t_{a}-\tau_{a}, q_{a}, t_{b}-\tau_{b}, q_{b}\right)=z^{\prime}=L_{\tau} z
\end{aligned}
$$

where $N_{a}$ and $N_{b}$ are the particle numbers of the respective subsystems.

At first thought, one might not expect a quantum theory to be invariant under $L_{\tau}$, because absolute time seems necessary to mediate the action-at-adistance of Schrödinger's equation, not to mention the more explicit nonlocality of Bohmian mechanics. Indeed, for the usual Schrödinger equation as well as for the GRW model and Bohmian mechanics it would appear that the multitime translation cannot be discussed at all because time appears in the wave function only as common (absolute) time.

But if the subsystems $a$ and $b$ are independent, i.e., if there is no interaction potential between the subsystems

$$
\begin{gathered}
V\left(q_{a}, q_{b}\right)=V_{a}\left(q_{a}\right)+V_{b}\left(q_{b}\right) \\
H=H_{a}+H_{b}, \quad H_{k}=-\frac{1}{2} \Delta_{k}+V_{k}, \quad k=a, b
\end{gathered}
$$




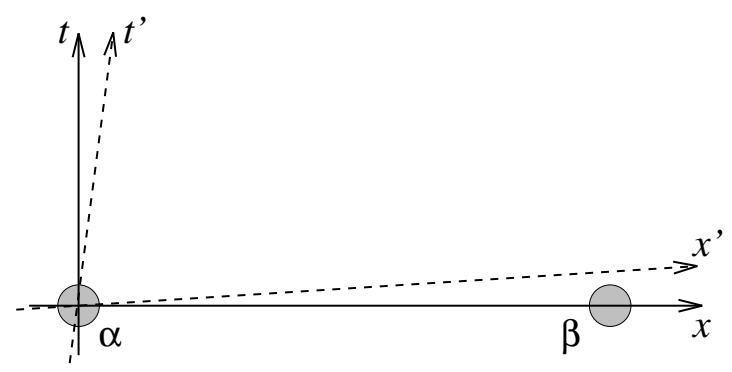

Figure 2: $\alpha$ and $\beta$ are two widely separated events. In the primed frame of reference, corresponding to a slowly moving observer, these two events are not simultaneous.

so that the Hamiltonians $H_{a}$ and $H_{b}$ commute, the Schrödinger evolution may be reformulated so that it becomes multitime translation invariant: From the ordinary one-time wave function $\psi_{t}=e^{-i H t} \psi_{0}=U_{t} \psi_{0}$ we define a two-time wave function $\psi\left(t_{a}, t_{b}\right) \in L^{2}\left(\mathbb{R}^{3 N_{a}}\right) \otimes L^{2}\left(\mathbb{R}^{3 N_{b}}\right) \cong L^{2}\left(\mathbb{R}^{3\left(N_{a}+N_{b}\right)}\right)$

$$
\psi\left(t_{a}, t_{b}\right)=e^{-i H_{a} t_{a}} e^{-i H_{b} t_{b}} \psi_{0}=U_{t_{a}}^{a} U_{t_{b}}^{b} \psi_{0}
$$

satisfying two separate Schrödinger equations

$$
i \frac{\partial \psi}{\partial t_{a}}=H_{a} \psi, \quad i \frac{\partial \psi}{\partial t_{b}}=H_{b} \psi
$$

This system of partial differential equations, with $\psi$ transforming in the obvious way,

$$
\psi(z)=\psi \circ L_{\tau}^{-1}\left(L_{\tau} z\right)=: \psi^{\prime}\left(z^{\prime}\right)
$$

is invariant under $L_{\tau}$. In particular, the unitary representation of the group of multitime translations is given by $U_{\tau}=U_{\tau_{a}}^{a} U_{\tau_{b}}^{b}$ :

$$
\psi^{\prime}=e^{-i H_{a} \tau_{a}} e^{-i H_{b} \tau_{b}} \psi=U_{\tau_{a}}^{a} U_{\tau_{b}}^{b} \psi=U_{\tau} \psi
$$

Note that in any frame of reference given by a particular synchronization of the subsystem times, i.e., where $t_{a}=s$ is simultaneous with $t_{b}=s+h$, the wave function in "frame $h$," which is given by $\psi_{t}^{h}=\psi(t, t+h)$ and recognized as $\psi_{t}^{h}=\psi^{\prime}(t, t)$ for a multitime shift by $\tau=(0, h)$ from the unprimed frame, satisfies the one-time Schrödinger equation.

It is also easy to see that the transition to a two-time wave function transforms the usual quantum measurement formalism into a multitime translation invariant form. We shall use here the Heisenberg picture for convenience as well as for analogy with relativistic quantum theory. Let $\psi=\psi_{0}=\psi(0,0)$ be the Heisenberg state of the system, and consider a sequence of observables $\left(M_{j}^{a}\right)_{1 \leq j \leq k}$ and $\left(M_{j}^{b}\right)_{1 \leq j \leq \ell}$, which are measured at times $t_{1}^{a}<\ldots<t_{k}^{a}$ resp. $t_{1}^{b}<\ldots<t_{\ell}^{b}$. Here $M_{j}^{a}$ acts only on system $a$, i.e., $M_{j}^{a}=O_{j}^{a} \otimes \mathbb{1}$ with observables $O_{j}^{a}$ on $L^{2}\left(\mathbb{R}^{3 N_{a}}\right)$, and $M_{j}^{b}$ acts only on system $b$, i.e., $M_{j}^{b}=\mathbb{1} \otimes O_{j}^{b}$ with 
observables $O_{j}^{b}$ on $L^{2}\left(\mathbb{R}^{3 N_{b}}\right)$. Thus the observables and the unitary evolution of system $a, M_{j}^{a}$ and $U_{t_{a}}^{a}=e^{-i H_{a} t_{a}}$, commute with the observables and the unitary evolution of system $b, M_{j}^{b}$ and $U_{t_{b}}^{b}=e^{-i H_{b} t_{b}}$ : for all $j, j^{\prime}, t_{a}, t_{b}$,

$$
\left[M_{j}^{a}, M_{j^{\prime}}^{b}\right]=0, \quad\left[M_{j}^{a}, U_{t_{b}}^{b}\right]=0, \quad\left[M_{j}^{b}, U_{t_{a}}^{a}\right]=0, \quad\left[U_{t_{a}}^{a}, U_{t_{b}}^{b}\right]=0 .
$$

We shall assume for simplicity that all the observables $M_{j}^{a}$ and $M_{j}^{b}$ have discrete spectrum and denote by $\pi_{j, \alpha}^{a}$ resp. $\pi_{j, \beta}^{b}$ the projection operator onto the eigenspace of $M_{j}^{a}$ resp. $M_{j}^{b}$ corresponding to the eigenvalue $\alpha$ resp. $\beta$. We introduce the Heisenberg operators

$$
\pi_{j, \alpha}^{a}\left(t_{a}\right):=U_{-t_{a}}^{a} \pi_{j, \alpha}^{a} U_{t_{a}}^{a} \quad \text { and } \quad \pi_{j, \beta}^{b}\left(t_{b}\right):=U_{-t_{b}}^{b} \pi_{j, \beta}^{b} U_{t_{b}}^{b},
$$

which, by (23), agree with the usual ones involving the full evolution $U_{t}=U_{t}^{a} U_{t}^{b}$. The joint probability for obtaining the measurement results $M_{1}^{a}=\alpha_{1}, \ldots$, $M_{k}^{a}=\alpha_{k}, M_{1}^{b}=\beta_{1}, \ldots, M_{\ell}^{b}=\beta_{\ell}$ is given by

$$
\begin{array}{r}
P\left(M_{1}^{a}=\alpha_{1}, \ldots, M_{k}^{a}=\alpha_{k}, M_{1}^{b}=\beta_{1}, \ldots, M_{\ell}^{b}=\beta_{\ell}\right) \\
\quad=\left\|\pi_{\ell, \beta_{\ell}}^{b}\left(t_{\ell}^{b}\right) \ldots \pi_{1, \beta_{1}}^{b}\left(t_{1}^{b}\right) \pi_{k, \alpha_{k}}^{a}\left(t_{k}^{a}\right) \ldots \pi_{1, \alpha_{1}}^{a}\left(t_{1}^{a}\right) \psi\right\|^{2} .
\end{array}
$$

Considering that under a multitime translation the Heisenberg operators transform as

$$
\begin{aligned}
& \pi_{j, \alpha}^{a}\left(t_{a}^{\prime}\right)=U_{-t_{a}^{\prime}}^{a} \pi_{j, \alpha}^{a} U_{t_{a}^{\prime}}^{a}=U_{\tau_{a}}^{a} \pi_{j, \alpha}^{a}\left(t_{a}\right) U_{-\tau_{a}}^{a}=U_{\tau} \pi_{j, \alpha}^{a}\left(t_{a}\right) U_{\tau}^{-1} \\
& \pi_{j, \beta}^{b}\left(t_{b}^{\prime}\right)=U_{-t_{b}^{\prime}}^{b} \pi_{j, \beta}^{b} U_{t_{b}^{\prime}}^{b}=U_{\tau_{b}}^{b} \pi_{j, \beta}^{b}\left(t_{b}\right) U_{-\tau_{b}}^{b}=U_{\tau} \pi_{j, \beta}^{b}\left(t_{b}\right) U_{\tau}^{-1}
\end{aligned}
$$

and the state transforms according to (22), one sees that the formula (24) is in fact multitime translation invariant. In particular, the predictions of the quantum measurement formalism are independent of the frame of reference. Thus the quantum mechanical measurement formalism for a system which consists of independent widely separated subsystems is multitime translation invariant.

Note also that the probability of obtaining the results $M_{i}^{a}=\alpha_{i}, M_{j}^{b}=\beta_{j}$ given the results $M_{1}^{a}=\alpha_{1}, \ldots, M_{i-1}^{a}=\alpha_{i-1}, M_{1}^{b}=\beta_{1}, \ldots, M_{j-1}^{b}=\beta_{j-1}$,

$$
\frac{\left\|\pi_{j, \beta_{j}}^{b}\left(t_{j}^{b}\right) \ldots \pi_{1, \beta_{1}}^{b}\left(t_{1}^{b}\right) \pi_{i, \alpha_{i}}^{a}\left(t_{i}^{a}\right) \ldots \pi_{1, \alpha_{1}}^{a}\left(t_{1}^{a}\right) \psi\right\|^{2}}{\left\|\pi_{j-1, \beta_{j-1}}^{b}\left(t_{j-1}^{b}\right) \ldots \pi_{1, \beta_{1}}^{b}\left(t_{1}^{b}\right) \pi_{i-1, \alpha_{i-1}}^{a}\left(t_{i-1}^{a}\right) \ldots \pi_{1, \alpha_{1}}^{a}\left(t_{1}^{a}\right) \psi\right\|^{2}}
$$

can be conveniently expressed as

$$
\left\|\pi_{j, \beta_{j}}^{b}\left(t_{j}^{b}\right) \pi_{i, \alpha_{i}}^{a}\left(t_{i}^{a}\right) \psi_{\mathrm{eff}}\right\|^{2}
$$

with the "collapsed wave function"

$$
\psi_{\mathrm{eff}}=\frac{\pi_{j-1, \beta_{j-1}}^{b}\left(t_{j-1}^{b}\right) \ldots \pi_{1, \beta_{1}}^{b}\left(t_{1}^{b}\right) \pi_{i-1, \alpha_{i-1}}^{a}\left(t_{i-1}^{a}\right) \ldots \pi_{1, \alpha_{1}}^{a}\left(t_{1}^{a}\right) \psi}{\left\|\pi_{j-1, \beta_{j-1}}^{b}\left(t_{j-1}^{b}\right) \ldots \pi_{1, \beta_{1}}^{b}\left(t_{1}^{b}\right) \pi_{i-1, \alpha_{i-1}}^{a}\left(t_{i-1}^{a}\right) \ldots \pi_{1, \alpha_{1}}^{a}\left(t_{1}^{a}\right) \psi\right\|} .
$$

(We find an analogous formula if we condition on a smaller initial segment.) 
Within this framework an EPR experiment can be described-the subsystems, while not explicitly interacting, are coupled by their common wave function $\psi\left(t_{a}, t_{b}\right)$ - and one can explicitly see, for this two-time yet orthodox model, that the EPR-Bell nonlocality does not demand the existence of a preferred frame of reference.

Despite the presence of EPR-correlations, these do not permit the transmission of "signals": From the results of measurements on system $a$ alone, one can draw no inference about the possible interventions on system $b$ - the kinds of experiments performed on system $b$. The crucial assumption responsible for this property is the commutativity (23). In axiomatic quantum field theory the analogue of this assumption, namely the commutativity of Heisenberg operators corresponding to measurements in spacelike separated regions, is one of the fundamental postulates, sometimes called "local commutativity" or "microscopic causality" (see for example [31]). It conveys that experiments in spacelike separated regions do not disturb each other, so that relativistic causality is not violated. However, it is important to recognize (as well as all too rare) that EPR and Bell have shown that the quantum correlations between observables for which "local commutativity" holds cannot in general be explained by a local theory!

Bell has shown that the GRW model can also be formulated in a multitime translation invariant manner ([1], Chapter 22). Bell's result is sometimes regarded as indicating that the GRW theory is superior to Bohmian mechanics with respect to the problem of finding a Lorentz invariant extension. In the next section we show that such a conclusion is perhaps unfounded.

\subsection{A multitime translation invariant Bohmian theory}

We formulate a multitime Bohmian theory that is invariant under multitime translation. Consider a system consisting of $n$ widely separated subsystems, as described in Section 3.1, with an $n$-time wave function satisfying (the analogue of) (21). As usual, we shall for simplicity put $n=2$. We shall denote again by $N_{a}$ and $N_{b}$ the particle numbers in the subsystems and put $N=N_{a}+$ $N_{b}$. The beables of the multitime Bohmian theory are first of all the usual beables of a Bohmian theory, namely the wave function, here the two-time wave function, and the trajectories of the particle configuration in the two subsytems, $Q_{a}(t)$ and $Q_{b}(t)$. The straightforward way to formulate a multitime translation invariant Bohmian theory for the evolution of these paths is to introduce as an additional beable a synchronization: a path in two-time $\mathbb{R}^{2}$, i.e., an equivalence class of maps $\left(T_{a}, T_{b}\right): \mathbb{R} \rightarrow \mathbb{R}^{2}, s \mapsto\left(T_{a}(s), T_{b}(s)\right)$ differing only in their parametrization. The synchronization together with the subsystem trajectories defines a synchronized $N$-path in configuration-space-time parametrized by $s$

$$
\left(T_{a}(s), Q_{a}(s), T_{b}(s), Q_{b}(s)\right)=: Z(s)
$$

with $Q_{a}(s) \equiv Q_{a}\left(T_{a}(s)\right), Q_{b}(s) \equiv Q_{b}\left(T_{b}(s)\right)$. We prescribe for the synchronized $N$-path the following guiding equation

$$
\frac{d T_{a}}{d s}=1, \quad \frac{d T_{b}}{d s}=1,
$$




$$
\frac{d Q_{a}}{d s}=v_{a}^{\psi}(Z), \quad \frac{d Q_{b}}{d s}=v_{b}^{\psi}(Z),
$$

with $v_{a}^{\psi}$ and $v_{b}^{\psi}$ given as usual by

$$
v_{a}^{\psi}=\operatorname{Im} \frac{\nabla_{q_{a}} \psi}{\psi}, \quad v_{b}^{\psi}=\operatorname{Im} \frac{\nabla_{q_{b}} \psi}{\psi}
$$

The Bohmian theory given by the Eqs. 21, 29, 20) does not have a preferred "frame of reference," and is obviously invariant under $L_{\tau}$, i.e., if $(\psi, Z)$ is a solution of (21, 25), then so is $\left(\psi^{\prime}, Z^{\prime}\right)=\left(\psi \circ L_{\tau}^{-1}, L_{\tau} \circ Z\right)$. The parameter $s$ labels the synchronization with respect to which the nonlocal interaction is mediated: The velocity of system $a$ at the parameter value $s$ depends, through $\psi\left(t_{a}, q_{a}, t_{b}, q_{b}\right)$, upon the configuration of the $a$-system at time $T_{a}(s)$-more precisely, upon $Q_{a}(s)$ and $T_{a}(s)$ - as well as on the configuration $Q_{b}(s)$ and the time $T_{b}(s)$ of the $b$-system corresponding to parameter value $s$. In particular, the velocity "field" is a functional of the two-time wave function at the appropriate times. Physical significance pertains only to the synchronized $N$-path $Z(\mathbb{R}) \subset$ $\mathbb{R}^{2+3 N}$, not to the particular parametrization determined by (25). Thus, just as with Eqn. (11), (25) is physically equivalent to all equations of the form $\frac{d Z}{d s}=A(Z)\left(1, v_{a}^{\psi}(Z), 1, v_{b}^{\psi}(Z)\right)$ with arbitrary positive functions $A$ on $\mathbb{R}^{2+3 N}$.

For the statistical analysis of this theory, it is natural to look for a distinguished measure. As a consequence of (21), we have the two identities which have the form of continuity equations

$$
\frac{\partial|\psi|^{2}}{\partial t_{a}}+\operatorname{div}_{q_{a}} j_{a}^{\psi}=0 \quad \text { or } \quad \operatorname{div}_{z_{a}} J_{a}^{\psi}=0
$$

and

$$
\frac{\partial|\psi|^{2}}{\partial t_{b}}+\operatorname{div}_{q_{b}} j_{b}^{\psi}=0 \quad \text { or } \quad \operatorname{div}_{z_{b}} J_{b}^{\psi}=0
$$

with $J_{k}^{\psi}=\left(|\psi|^{2}, j_{k}^{\psi}\right)$ and $j_{k}^{\psi}=|\psi|^{2} v_{k}^{\psi}=\operatorname{Im}\left(\psi^{*} \nabla_{k} \psi\right), k=a, b$. By analogy with the statistical analysis of the usual Bohmian mechanics, it might at first glance seem appropriate to seek a stationary measure for $Z$, i.e., for the dynamical system given by Eqs. (25, 26). The continuity equation for this dynamical system, for a (continuously differentiable) density $f: \mathbb{R} \times \mathbb{R}^{2+3 N} \rightarrow \mathbb{R}$,

$$
\frac{\partial f}{\partial s}+\operatorname{div}_{z_{a}}\left(f w_{a}^{\psi}\right)+\operatorname{div}_{z_{b}}\left(f w_{b}^{\psi}\right)=0
$$

with $w_{k}^{\psi}:=\left(1, v_{k}^{\psi}\right)$, is, by (27) and (28), solved (trivially) by $f=|\psi|^{2}$, which is stationary with respect to the synchronization parameter $s$.

Hence $|\psi|^{2}$ is certainly a distinguished measure on the space $\mathbb{R}^{2+3 N}$ of initial values for Eqs. (25). But it is not normalizable (by unitarity); moreover, for the dynamical system given by Eqs. (25, 26) there can be no density, normalizable on $\mathbb{R}^{2+3 N}$, that is stationary with respect to the evolution parameter $s$ - since a stationary measure for $Z$ yields a stationary marginal measure for $T_{a}$, and by the first of Eqs. (25) all stationary measures for $T_{a}$ must be proportional to the 
Lebesgue measure on $\mathbb{R}$. In this regard it is also important to recognize that a general probability density $f(s)$ satisfying (29), while defining a probability measure on $N$-paths, does not itself directly correspond to any clear statistical property of this ensemble of $N$-paths, such as the distributions of crossings discussed in Section 2 .

Recall now that $s$ labels the synchronization, and recall as well the suggestion that "quantum equilibrium" should hold on "simultaneity surfaces" [21]. Thus we proceed as follows: We first fix initial values for the subsystem times $T_{a}(0)=: s_{0}$ and $T_{b}(0):=s_{0}+h$. Then the evolution equations for $T_{a}$ and $T_{b}$ may be solved to obtain $T_{a}(s)=s_{0}+s$ and $T_{b}(s)=s_{0}+h+s$. The constant of motion $h=T_{b}(0)-T_{a}(0)\left(=T_{b}(s)-T_{a}(s)\right.$ for all $\left.s\right)$ defines the synchronization-the velocity of system $a$ at a time $t_{a}$ depends upon the configuration $Q_{b}$ at time $t_{b}=t_{a}+h$. (As it happens, to this fixed synchronization we may associate a (Lorentz) frame of reference in which the interaction between the systems is "instantaneous." However, this associated frame is merely a convenience, one that for more than four subsystems it would typically be impossible to retain.)

Now the subsystem times in Eqn. (25) may be eliminated: With

$$
\psi^{h}\left(s, q_{a}, q_{b}\right):=\psi\left(T_{a}(s), q_{a}, T_{b}(s), q_{b}\right)=\psi\left(s_{0}+s, q_{a}, s_{0}+h+s, q_{b}\right)
$$

one obtains

$$
\begin{aligned}
& \frac{d Q_{a}}{d s}=v_{a}^{\psi^{h}}\left(s, Q_{a}(s), Q_{b}(s)\right), \\
& \frac{d Q_{b}}{d s}=v_{b}^{\psi^{h}}\left(s, Q_{a}(s), Q_{b}(s)\right) .
\end{aligned}
$$

This is the usual Bohmian mechanics relative to the synchronization given by $h$; we have the continuity equation

$$
\frac{\partial \rho^{h}}{\partial s}+\operatorname{div}_{q_{a}}\left(\rho^{h} v_{a}^{\psi^{h}}\right)+\operatorname{div}_{q_{b}}\left(\rho^{h} v_{b}^{\psi^{h}}\right)=0,
$$

and the density $\rho^{h}=\left|\psi^{h}\right|^{2}$ is "equivariant," i.e., if $\rho^{h}\left(s_{0}\right)=\left|\psi^{h}\left(s_{0}\right)\right|^{2}$ for some $s=s_{0}$, then $\rho^{h}(s)=\left|\psi^{h}(s)\right|^{2}$ for all $s$. For $\psi^{h}(s) \in L^{2}\left(\mathbb{R}^{3 N}\right)$, this density is normalizable, and gives the distribution of crossings of any hypersurface corresponding to the times $T_{a}(s)$ and $T_{b}(s)$ for the ensemble of $N$-paths defined by $\psi^{h}$.

\subsection{Hardy's experiment in multitime translation invariant Bohmian theory}

We describe now the particle trajectories in Hardy's experiment for the multitime translation invariant Bohmian theory given by Eqs. (21, 25, 26) (with $N_{a}=N_{b}=1$ and the Stern-Gerlach magnets treated, as usual, as external fields). We prepare a system of two particles in the quantum state $\psi_{\text {Hardy }}$ (4). After the particles are widely separated from each other, we perform Hardy's experiment, cf. Figure 1, focusing on the part of the experiment in which the particles run through the Stern-Gerlach magnets $A_{z}, B_{z}$, cf. Figure 3 . 

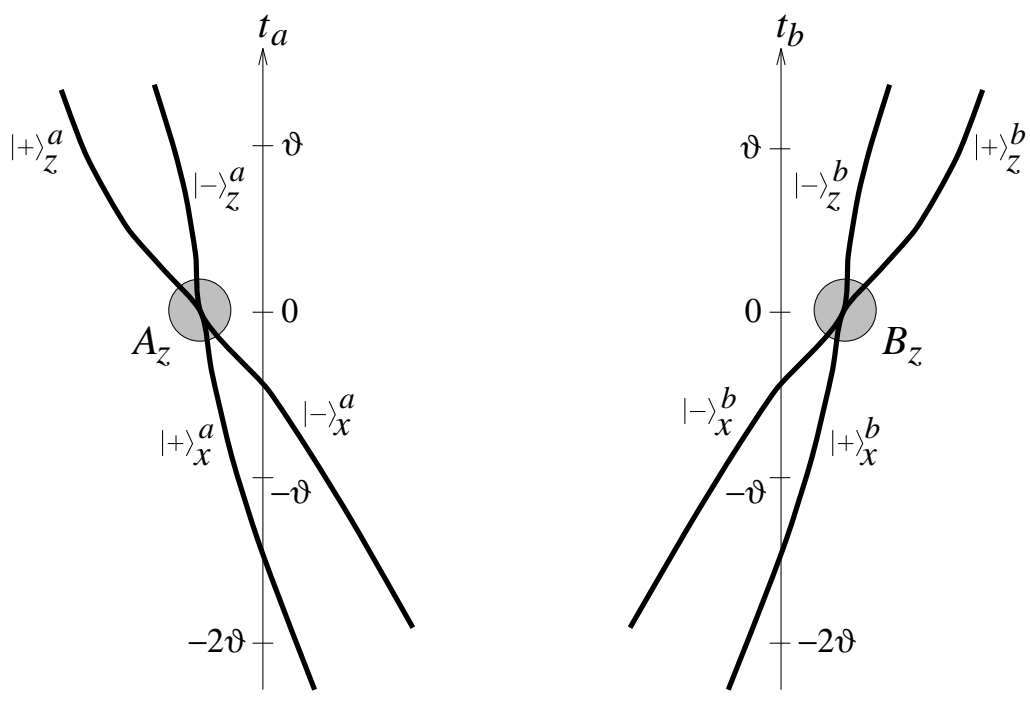

Figure 3: Hardy's experiment in multitime formalism.

First we describe the development of the synchronized paths for initial values of the subsystem times $T_{a}(0)=-\vartheta$ and $T_{b}(0)=h^{I I}=-2 \vartheta$ for a $\vartheta>0$, not too large, referring to scales for the subsystem times $t_{a}$ and $t_{b}$ as defined in Figure 3. This gives a synchronization corresponding to the frame $I I$ in Figure 1. Consider those two-paths for which at the value $s=0$ of the synchronization parameter, particle $a$ is located in supp $|+\rangle_{x}^{a}$ and particle $b$ is located in $\operatorname{supp}|+\rangle_{x}^{b}$. Demanding $\rho^{h^{I I}}(0)=\left|\psi^{h^{I I}}(0)\right|^{2}$, these are $1 / 12$ of all two-paths. After particle $a$ has gone through the apparatus $A_{z}$, it must be located in $\operatorname{supp}|-\rangle_{z}^{a}$ since e.g. $\rho^{h^{I I}}(3 \vartheta / 2)=\left|\psi^{h^{I I}}(3 \vartheta / 2)\right|^{2}$, cf. Eqn. (13). After particle $b$ has run through the apparatus $B_{z}$, it must be located in supp $|+\rangle_{z}^{b}$ since e.g. $\rho^{h^{I I}}(5 \vartheta / 2)=\left|\psi^{h^{I I}}(5 \vartheta / 2)\right|^{2}$, cf. Eqn. (15). This course of the particle paths is displayed in Figure 4 , top.

Now consider the same experiment with different initial values for the subsystem times: $T_{a}(0)=-2 \vartheta$ and $T_{b}(0)=-\vartheta$, so that $h=h^{I I I}=\vartheta$, a synchronization corresponding to the frame $I I I$ in Figure 1. Again consider those two-paths for which at the value $s=0$ of the synchronization parameter, particle $a$ is located in supp $|+\rangle_{x}^{a}$ and particle $b$ is located in supp $|+\rangle_{x}^{b}$. Demanding $\rho^{h^{I I I}}(0)=\left|\psi^{h^{I I I}}(0)\right|^{2}$, these are $1 / 12$ of all two-paths. After particle $b$ has gone through $B_{z}$, it must be located in supp $|-\rangle_{z}^{b}$ since e.g. $\rho^{h^{I I I}}(3 \vartheta / 2)=$ $\left|\psi^{h^{I I I}}(3 \vartheta / 2)\right|^{2}$, cf. Eqn. (14). After particle $a$ has run through the apparatus $A_{z}$, it must be located in supp $|+\rangle_{z}^{a}$ since e.g. $\rho^{h^{I I I}}(5 \vartheta / 2)=\left|\psi^{h^{I I I}}(5 \vartheta / 2)\right|^{2}$, cf. Eqn. (15). This course of the particle paths is displayed in Figure 14, bottom.

In neither case does the distribution of crossings by the two-paths of a hypersurface corresponding to the other synchronization agree for all parameter values $s$ with the corresponding $\left|\psi^{h}(s)\right|^{2}$. In the first case, $h=h^{I I}=-\vartheta$, the two-paths run through supp $|+\rangle_{x}^{a} \times \operatorname{supp}|+\rangle_{z}^{b}$ when they cross a suitable 

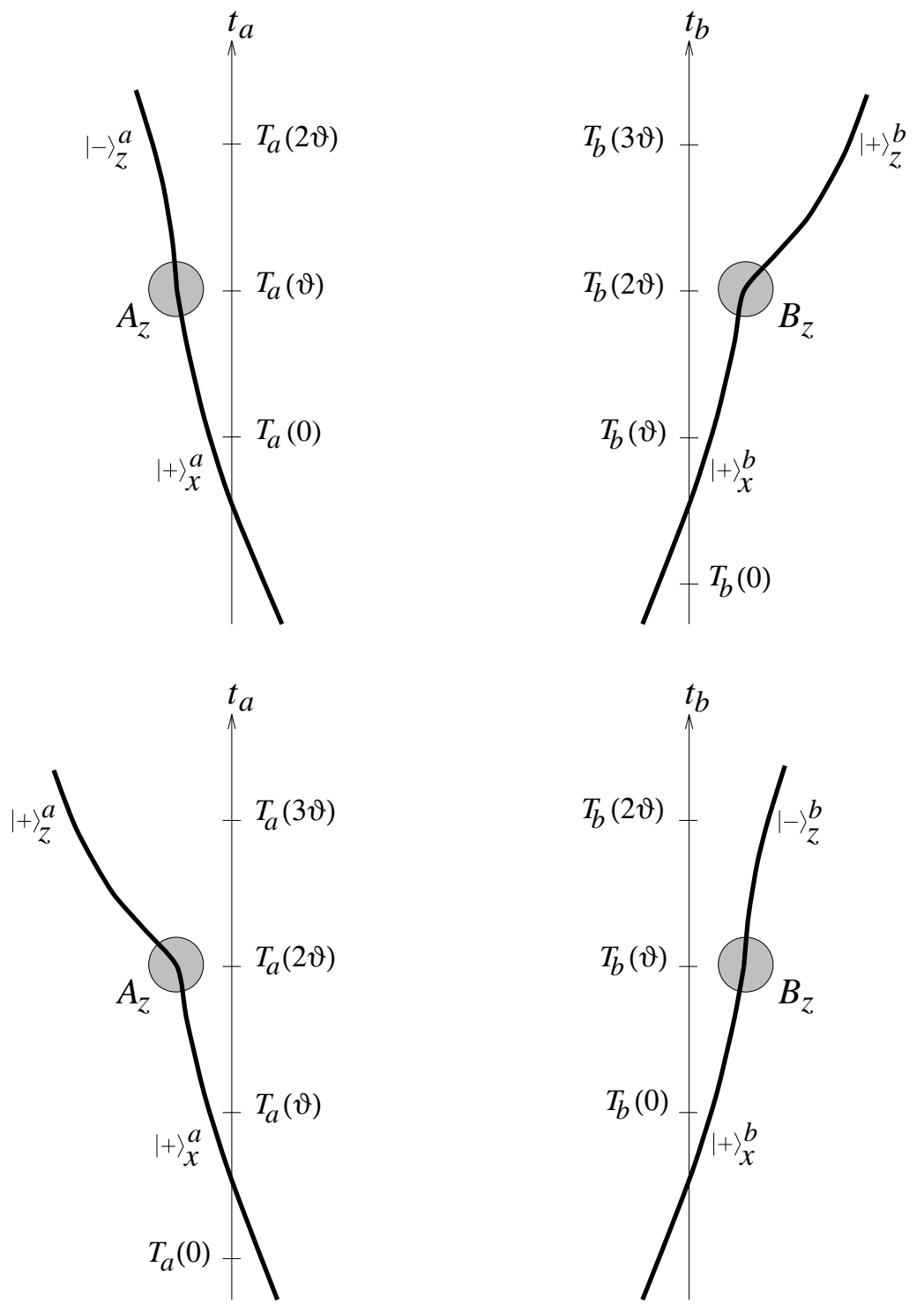

Figure 4: Course of some synchronized two-paths according to the multitime translation invariant Bohmian theory in Hardy's experiment for initial values of subsystem times corresponding to the synchronization II (top) resp. III (bottom). 
hypersurface corresponding to frame $I I I$, even though the wave function $\psi^{h}$ is orthogonal to $|+\rangle_{x}^{a}|+\rangle_{z}^{b}$, cf. Eqn. (14). Analogously, in the second case, $h=$ $h^{I I I}=\vartheta$, the two-paths run through $\operatorname{supp}|+\rangle_{z}^{a} \times \operatorname{supp}|+\rangle_{x}^{b}$ when they cross a suitable hypersurface corresponding to frame $I I$, even though the wave function $\psi^{h}$ is orthogonal to $|+\rangle_{z}^{a}|+\rangle_{x}^{b}$, cf. Eqn. (13).

Finally we explain why, despite the fact that the two-paths (occasionally) are in regions where the wave function vanishes, no violations of the quantum mechanical predictions would be experimentally observed. If an actual experiment-involving, for example, the insertion of photographic plates into the paths of the particles - were performed, the influence of this apparatus on the future evolution of the complete system would have to be taken into account. This can conveniently be accomplished, in a manner analogous to what is done in ordinary Bohmian mechanics, by suitably collapsing the wave function $\psi^{h}$ upon measurement. Suppose, for example, that we attempt to detect what quantum mechanically should be impossible, namely the two-paths running through $\operatorname{supp}|+\rangle_{x}^{a} \times \operatorname{supp}|+\rangle_{z}^{b}$, which we have just seen has positive probability for synchronization $I I$, at least when no detection is attempted. We might do this by inserting a detector in the path corresponding to $\operatorname{supp}|+\rangle_{x}^{a}$, say at a position corresponding to $s=0$, as well as in the path corresponding to $\operatorname{supp}|+\rangle_{z}^{b}$. Then with the synchronization $I I$, the wave function $\psi^{h^{I I}}$ collapses at the synchronization parameter value $s=0$, when particle $a$ is found in $\operatorname{supp}|+\rangle_{x}^{a}$, to

$$
\psi_{a_{x}=+1}^{h^{I I}}(s=0)=\frac{1}{\sqrt{2}}|+\rangle_{x}^{a}\left(|+\rangle_{x}^{b}-|-\rangle_{x}^{b}\right) \stackrel{U_{5 \vartheta / 2}}{\longrightarrow}|+\rangle_{x}^{a}|-\rangle_{z}^{b},
$$

and the future evolution of particle $b$ changes drastically from what it would have been like had there been no measurement or collapse: after having gone through apparatus $B_{z}$ it no longer runs into the $|+\rangle_{z}^{b}$ channel, but rather into the $|-\rangle_{z}^{b}$ channel! Analogous things happen with the synchronization III and the opposite measurements.

\section{Reflections on Lorentz invariance and statistical transparency}

Concerning the model of Section 3.2, we have just alluded to the fact that, just as for ordinary Bohmian mechanics, from the quantum equilibrium hypothesis that the actual distribution of crossings $\rho^{h}=\left|\psi^{h}\right|^{2}$, one can derive the quantum mechanical measurement formalism - which, as shown in Section 3.1, is multitime translation invariant and moreover does not even depend upon the quantity $h$. We thus have, with regard to our multitime Bohmian model, three levels of description: the microscopic dynamical level, given by (21,25), which is multitime translation invariant; the statistical mechanical level, given by the quantum equilibrium hypothesis, which is, in precisely the same way, also multitime translation invariant - despite the results of Section 2; and the observational level given by the quantum measurement formalism, which is also apparently multitime translation invariant. 
There is, however, an important difference between the relativistic characters of these levels: the latter level might be regarded as more fully relativistic than the first two, which achieve their invariance through the incorporation of the additional structure provided by the synchronization. It might be argued that such a structure violates the spirit of relativity [4, 12], and regardless of whether or not we agree with this, it must be admitted that achieving relativistic invariance in a realistic (i.e., precise) version of quantum theory without the invocation of such structure seems much more difficult. Hence Bell's excitement about his version of the model of GRW ([1], Chapter 22). (It must also be admitted that a somewhat unpleasant implication of the situation just described is that this synchronization structure - which after all comprises a radical addition to physics - is, in the model under consideration here, completely unobservable! See also 涵.)

Indeed, any theory can be made trivially Lorentz invariant (or invariant under any other space-time symmetry) by the suitable incorporation of additional structure, for example as given by the specification of a Lorentz frame $\Lambda_{0}$ as part of the state description. It seems rather clear that this example, while Lorentz invariant, does not possess what Bell has called "serious Lorentz invariance," a notion, however, that it is extremely difficult to make precise in an adequate way [1].

The Bohmian model (25) immediately suggests a genuinely (though perhaps not seriously) Lorentz invariant Bohmian theory: For $N$ particles, the beables are a multitime wave function and a synchronized $N$-path, i.e., an equivalence class of maps $\left(X_{1}, \ldots, X_{N}\right): \mathbb{R} \rightarrow \mathbb{R}^{4 N}, s \mapsto\left(X_{1}(s), \ldots, X_{N}(s)\right)$ differing only in their parametrization. The synchronized $N$-path satisfies the guiding equation

$$
\frac{d X_{k}}{d s}=v_{k}\left(X_{1}(s), \ldots, X_{N}(s)\right), \quad k=1, \ldots, N,
$$

where the $v_{k}$ are suitable 4 -vector fields, on $\mathbb{R}^{4 N}$, determined by the multitime wave function. As with (1) and (25), the fact that only the synchronized $N$-path and not the parametrization determined by a particular $v_{k}$ has beable status implies that all equations of the form $\frac{d X_{k}}{d s}=a\left(X_{1}, \ldots, X_{N}\right) v_{k}\left(X_{1}, \ldots, X_{N}\right)$ with an arbitrary positive function $a$ on $\mathbb{R}^{4 N}$ are physically equivalent.

More concretely, one may consider a Lorentz invariant multitime BohmDirac theory: the wave function $\psi=\psi\left(x_{1}, \ldots, x_{N}\right)$ satisfies $N$ Dirac equations analogous to (21), and $v_{k}$ may for example be chosen to be

$$
v_{k}^{\mu}=\bar{\psi} \gamma_{k}^{\mu} \psi
$$

\footnotetext{
${ }^{6}$ Consider a theory specifying the set $\mathcal{L}$ (THE LAW) of possible decorations $\xi$ of spacetime and assume that the Lorentz group acts naturally on any $\xi$ and thus on $\mathcal{L}$. This theory, demanding that $\xi \in \mathcal{L}$, will be Lorentz invariant if $\Lambda \mathcal{L}=\mathcal{L}$ for any Lorentz transformation $\Lambda$. Suppose this is not true. We may then enlarge the original theory by replacing $\xi$ by $\hat{\xi} \equiv\left(\xi, \Lambda_{0}\right)$ and the law $\mathcal{L}$ by $\hat{\mathcal{L}}$ defined by stipulating that $\left(\xi, \Lambda_{0}\right)=\hat{\xi} \in \hat{\mathcal{L}} \Leftrightarrow \xi \in \Lambda_{0} \mathcal{L}$. (The original theory thus corresponds to $\Lambda_{0}=I$.) Then $\hat{\mathcal{L}}$ is trivially Lorentz invariant: For any Lorentz transformation $\Lambda$ we have that $\Lambda \hat{\xi}=\left(\Lambda \xi, \Lambda \Lambda_{0}\right) \equiv\left(\xi^{\prime}, \Lambda_{0}{ }^{\prime}\right) \equiv \hat{\xi}^{\prime}$, so that $\hat{\xi} \in \hat{\mathcal{L}} \Rightarrow \Lambda \xi \in \Lambda \Lambda_{0} \mathcal{L} \Rightarrow \xi^{\prime} \in \Lambda_{0}^{\prime} \mathcal{L} \Rightarrow \hat{\xi}^{\prime} \in \hat{\mathcal{L}}$.
} 
with $\bar{\psi}=\psi^{\dagger}\left(\gamma^{0} \otimes \ldots \otimes \gamma^{0}\right)=\psi^{\dagger} \gamma_{1}^{0} \ldots \gamma_{N}^{0}$ and $\gamma_{k}^{\mu}=1 \mathbb{1} \otimes \ldots \otimes \mathbb{1} \otimes \gamma^{\mu} \otimes \mathbb{1} \otimes \ldots \otimes \mathbb{1}$, the $\gamma^{\mu}$ at the $k$-th of the $N$ places. We shall discuss such a model in a subsequent work. Just as with the model of Section 3, models of the form (31), because of the nonlocal interaction along the synchronization, have the possibility of properly describing quantum nonlocality as exhibited, for example, by an EPR experiment. This is in contrast with the local model of Squires [32], which is based on what might be called a local light-cone synchronization. While Squires formulates his model for the nonrelativistic Schrödinger equation, he could as well have considered a multitime Dirac model with a local light-cone synchronization to obtain a model that is completely Lorentz invariant - and completely local.

Some readers may be wondering why we have analysed the nonrelativistic multitime Bohmian theory in detail in Section 3 instead of starting right away with (31, 32) or with the multitime Bohm-Dirac theory

$$
\frac{d T_{k}}{d s}=\psi^{\dagger} \psi, \quad \frac{d \mathbf{Q}_{k}}{d s}=\psi^{\dagger} \boldsymbol{\alpha}_{k} \psi
$$

with $X_{k}=\left(T_{k}, \mathbf{Q}_{k}\right), k=1 \ldots N$, and $\alpha_{k}^{i}=\mathbb{1} \otimes \ldots \otimes \mathbb{1} \otimes \alpha^{i} \otimes \mathbb{1} \otimes \ldots \otimes \mathbb{1}=$ $\gamma_{k}^{0} \gamma_{k}^{i}$. This theory arises from Bohm's theory (3) for $N$ Dirac particles $\mathbf{v}_{k}=$ $\frac{\psi^{\dagger} \boldsymbol{\alpha}_{k} \psi}{\psi^{\dagger} \psi}=\frac{\mathbf{j}_{k}}{\rho}$ by introducing a dynamical synchronization, and it agrees for $N=$ 1 with (31, 32). These models might suggest that the reconciliation of statistical transparency and Lorentz invariance is at hand. However, for $N>1$ (33) is not Lorentz invariant, because - unlike (32) - $\left(\psi^{\dagger} \psi, \psi^{\dagger} \boldsymbol{\alpha}_{k} \psi\right)$ is not a 4 -vector. On the other hand, (32) is not statistically transparent because - unlike (33) - the (reparametrization invariant) configuration space velocity $v_{k}^{i} / v_{k}^{0}$ arising from (32) is not of the form $\mathbf{j}_{k} / \rho$ for $N>1$. Thus, for the Lorentz invariant model (31, 32) equivariance does not hold in any obvious way and hence, since there is in general no reason that quantum equilibrium should hold with respect to any reasonable family of hypersurfaces, the canonical statistical analysis cannot be performed and the question of the extent of its agreement with standard quantum theory becomes rather delicate. 1

There is another important difference between (32) and (33). To appreciate this consider the system

$$
\frac{d T_{k}}{d s}=1, \quad \frac{d \mathbf{Q}_{k}}{d s}=\mathbf{v}_{k}\left(X_{1}(s), \ldots, X_{N}(s)\right)
$$

with $\mathbf{v}_{k}=\frac{\psi^{\dagger} \boldsymbol{\alpha}_{k} \psi}{\psi^{\dagger} \psi}$. Here $\left(T_{k}(s)\right)$ is entirely determined by $\left(T_{k}(0)\right)$ and the statistical analysis of this theory may be developed as in Section 3.2 for the multitime Bohmian theory, merely replacing $|\psi|^{2}$ by $\psi^{\dagger} \psi$. With (32), however, the equations for the evolution of the synchronized particle times

$$
\frac{d T_{k}}{d s}=\left(\bar{\psi} \gamma_{k}^{0} \psi\right)\left(X_{1}(s), \ldots, X_{N}(s)\right)
$$

\footnotetext{
${ }^{7}$ This absence of statistical transparency is similarly also the case for the local model of Squires 32].
} 
imply that in general $\left(T_{k}(s)\right)$ depends upon the (initial) positions of the particles as well as on $\left(T_{k}(0)\right)$, and it is difficult to see how one could begin any statistical analysis even if the velocity field were otherwise somehow of a suitable form. Now it might appear that we should have the same difficulty with (33); however the theory (33) is equivalent to (34) since the respective vector fields differ by a real-valued function on $\mathbb{R}^{4 N}$ and hence define the same synchronized $N$-paths. Thus it turns out that (33) is statistically transparent - or at least statistically translucent. We shall take up these questions in a subsequent paper.

Observe that if the 4 -vectors $v_{k}$ are 4 -velocities $\left(v_{k \mu} v_{k}^{\mu}=1\right)$, the synchronization implied by (31), which in this case is according to proper time parametrization, reduces in the nonrelativistic limit to the first set of Eqs. (25). 3. Whatever reservations we may have concerning models such as we've been discussing, a synchronization by proper time seems to us entirely compatible with serious Lorentz invariance, at least for a pair of particles having a common origin in a single event.

The requirement that a Bohmian theory be Lorentz invariant without the incorporation of such additional structure as a dynamical synchronization places a very strong constraint on, say, the vector field defining the law of motion (in a particular frame), or, what amounts to pretty much the same thing, on the wave function of the system - and might be expressed via a suitable fixed-point equation for this wave function. It seems extremely likely that the set of wave functions satisfying such an equation is very small, far smaller than the families of wave functions we normally consider for the set of possible initial states of a quantum system. However, if, as is widely believed, we accept that from a cosmological perspective there should be a unique wave function (for example, the Wheeler-de Witt wave function or the Hartle-Hawking wave function) of the universe, this very fact might well be a virtue rather than a vice!

\section{Acknowledgements}

This work was supported in part by the DFG, by NSF Grant No. DMS-9504556, and by the INFN.

\footnotetext{
${ }^{8}$ Note that $\bar{\psi} \gamma_{k}^{\mu} \psi$ need not in general be everywhere timelike and thus $v_{k}(32)$ cannot in general be normalized. However, one can find a simple reparametrization $v_{k}=\frac{\bar{\psi} \gamma_{k} \psi}{\bar{\psi} \psi}$ such that the $v_{k}$ are approximate 4 -velocities for "large $c$ ": Writing

$$
\psi\left(x_{1}, \ldots, x_{N}\right)=\sum_{i} \varphi_{i}\left(x_{k}\right) \chi_{i}\left(x_{1}, \ldots, x_{k-1}, x_{k+1}, \ldots, x_{N}\right)
$$

and noting that in the nonrelativistic limit the last two components of $\varphi_{i}$ in the standard representation become much smaller than the first two, one sees that in the nonrelativistic limit $d T_{k} / d s=\bar{\psi} \gamma_{k}^{0} \psi / \bar{\psi} \psi \approx 1$ and the space components of $v_{k}$ become small. Thus in the nonrelativistic limit the theory (32) implies a synchronization that can be (re)expressed in the form (25) (first equations). (Concerning the reparametrization by $\bar{\psi} \psi$ [and that by $\psi^{\dagger} \psi$ for the relation between (33, 34)], one may convince oneself that not only the multiplication of the velocity field by a positive function, but typically even by a fuction that has zeros or changes sign will yield an equivalent theory.)
} 


\section{References}

[1] J.S. Bell, Speakable and Unspeakable in Quantum Mechanics (Cambridge University Press, Cambridge, 1987).

[2] J.S. Bell, Physics World 3, 33 (August 1990).

[3] D.Z. Albert, Quantum Mechanics and Experience (Harvard University Press, Cambridge, 1992).

[4] T. Maudlin, in Bohmian Mechanics and Quantum Theory: An Appraisal, edited by J.T. Cushing, A. Fine, S. Goldstein (Kluwer Academic, Dordrecht, to appear).

[5] D. Bohm, Phys. Rev. 85, 166 (1952); 85, 180 (1952).

[6] D. Dürr, S. Goldstein, and N. Zanghì, J. Stat. Phys. 67, 843 (1992).

[7] G.C. Ghirardi, A. Rimini, and T. Weber, Phys. Rev. D 34, 470 (1986).

[8] D. Bohm, Prog. Theor. Phys. 9, 273 (1953).

[9] D. Bohm and B.J. Hiley, The Undivided Universe: An Ontological Interpretation of Quantum Theory (Routledge, London, 1993).

[10] P.R. Holland, The Quantum Theory of Motion (Cambridge University Press, Cambridge, 1993).

[11] A. Einstein, B. Podolsky, and N. Rosen, Phys. Rev. 47, 777 (1935).

[12] T. Maudlin, Quantum Non-locality and Relativity (Blackwell, Cambridge, 1994).

[13] J.A. Wheeler and R.P. Feynman, Rev. Mod. Phys. 21, 425 (1949).

[14] D. Bohm, B.J. Hiley, and P.N. Kaloyerou, Phys. Rep. 144, 321 (1987).

[15] L. Hardy, Phys. Rev. Lett. 68, 2981 (1992).

[16] L. Hardy, Phys. Rev. Lett. 71, 1665 (1993).

[17] S. Goldstein, Phys. Rev. Lett. 72, 1951 (1994).

[18] L. Hardy, Phys. Rev. Lett. 73, 2279 (1994).

[19] K. Berndl and S. Goldstein, Phys. Rev. Lett. 72, 780 (1994).

[20] L. Hardy, Phys. Rev. Lett. 72, 781 (1994).

[21] D. Dürr, S. Goldstein, and N. Zanghì, in Stochastic Processes, Physics and Geometry, edited by S. Albeverio, G. Casati, U. Cattaneo, D. Merlini, and R. Moresi (World Scientific, Singapore, 1990), p. 374.

[22] G.C. Ghirardi, R. Grassi, J. Butterfield, and G.N. Fleming, Found. Phys. 23, 341 (1993). 
[23] L. Hardy and E.J. Squires, Phys. Lett. A 168, 169 (1992).

[24] J.S. Bell, in Themes in Contemporary Physics II: Essays in Honor of Julian Schwinger's 70th Birthday, edited by S. Deser and R.J. Finkelstein (World Scientific, Singapore, 1989), p. 1.

[25] G. Wentzel, Quantum Theory of Fields (Interscience, New York, 1949).

[26] T.M. Samols, J. Stat. Phys. 80, 793 (1995).

[27] J.F. Clauser, M.A. Horne, A. Shimony, and R.A. Holt, Phys. Rev. Lett. 23, 880 (1969).

[28] D.M. Greenberger, M. Horne, and A. Zeilinger, in Bell's Theorem, Quantum Theory, and Conceptions of the Universe, edited by M. Kafatos (Kluwer Academic, Dordrecht, 1989), p. 69.

[29] N.D. Mermin, Phys. Rev. Lett. 65, 3373 (1990).

[30] M. Daumer, D. Dürr, S. Goldstein, and N. Zanghì, On the role of operators in quantum theory, in preparation.

[31] R.F. Streater and A.S. Wightman, PCT, Spin and Statistics, and All That (Addison-Wesley, Redwood City, 1989).

[32] E. Squires, Phys. Lett. A 178, 22 (1993). 